The Journal of Engineering and Exact Sciences - jCEC, Vol. 06 N. 02 (2020)

journal homepage: https://periodicos.ufv.br/ojs/jcec

doi: 10.18540/jcecvl6iss2pp0189-0192

OPEN ACCESS - ISSN: 2527-1075

\title{
COMBINATORIAL TECHNIQUE FOR OPTIMIZING THE COMBINATION
}

Chinnaraji Annamalai ${ }^{1, *}$, Junzo Watada ${ }^{2}$, Said Broumi $^{3}$ and Vishnu Narayan Mishra ${ }^{4}$

${ }^{1}$ Department of Management, Indian Institute of Technology,

Kharagpur, Paschim Medinipur, West Bengal - 721302, India

ORCID: https://orcid.org/0000-0002-0992-2584

${ }^{2}$ Graduate School of Information, Production and Systems, Waseda University, 1-104 Totsukamachi, Shinjuku-ku, Tokyo, 69-8050, Japan

ORCID: https://orcid.org/0000-0002-3322-2086

${ }^{3}$ Laboratory of Information Processing, Faculty of Science Ben M'Sik, University Hassan II, B.P 7955, Sidi Othman, Casablanca, Morocco ORCID: https://orcid.org/0000-0002-1334-5759

${ }^{4}$ Department of Mathematics, Indira Gandhi National Tribal University, Lalpur, Amarkantak, Anuppur, Madhya Pradesh, India

ORCID: https://orcid.org/0000-0002-2159-7710

"Corresponding author. Indian Institute of Technology, Kharagpur, Paschim Medinipur, West Bengal, India, Phone: +91 3222282155

e-mail addressl: anna@iitkgp.ac.in (Chinnaraji Annamalai).

A R T I C L E IN F O

Article history:

Received 2020-05-10

Accepted 2020-06-17

Available online 2020-06-17

keywords

Optimized Combination

Combinatorics

Counting technique

Binomial coefficient
A B S T R A C T

This paper presents an innovative computing method and models for optimizing the combination defined in combinatorics. The optimized combination has been derived from the iterative computation of multiple geometric series and summability by specialized approach. The optimized combinatorial technique has applications in science, engineering and management. In this paper, several properties and consequences on the innovative optimized combination has been introduced that are useful for scientific researchers who are solving scientific problems and meeting today's challenges. 


\section{INTRODUCTION}

Combinatorics is a collection of various counting techniques or methods and models and has many applications in science, technology, and management. In the research paper, optimized combination of combinatorics is introduced that is useful for scientific researchers who are solving scientific problems and meeting today's challenges.

\section{OPTIMIZED COMBINATION}

This optimized combination is derived from the iterative computations [Annamalai et al., 2018, 2019, 2020] of multi-geometric series and summability as follows

$\sum_{i_{1}=0}^{n-1} \sum_{i_{2}=i_{1}}^{n-1} \sum_{i_{3}=i_{2}}^{n-1} \ldots \ldots \sum_{i_{n}=i_{n-1}}^{n-1} x^{i_{n}}=\sum_{i=0}^{n-1} V_{i}^{p} x^{i},(p \in N \& 1 \leq p \leq n-1)$

Where $V_{\mathrm{i}}^{\mathrm{p}}$ is a binomial coefficient and its mathematical expressions are given below:

$V_{i}^{p}=\frac{(i+1)(i+2)(i+3) \ldots(i+p)}{p !} \quad(1 \leq p \leq n-1)$.

$V_{i-k}^{p}=\frac{(i-k+1)(i-k+2)(i-k+3) \ldots(i-k+p)}{p !}$.

Let us prove the equation (A) using the multiple geometric series.

$\sum_{i_{1}=0}^{n-1} \sum_{i_{2}=i_{1}}^{n-1} x^{i_{2}}=\sum_{i_{2}=0}^{n-1} x^{i_{2}}+\sum_{i_{2}=1}^{n-1} x^{i_{2}}+\sum_{i_{2}=2}^{n-1} x^{i_{2}}+\cdots+\sum_{i_{2}=n-1}^{n} x^{i_{2}}=\sum_{i=0}^{n-1} \frac{(i+1)}{1 !} x^{i}=\sum_{i=0}^{n-1} V_{i}^{1} x^{i}$

where $\sum_{i_{2}=0}^{n-1} x^{i_{2}}+\sum_{i_{2}=1}^{n-1} x^{i_{2}}+\sum_{i_{2}=2}^{n-1} x^{i_{2}}+\cdots+\sum_{i_{2}=n-1}^{n} x^{i_{2}}=1+2 x+3 x^{2}+\cdots+\frac{n}{1 !} x^{n-1}$.

$\sum_{i_{1}=0}^{n-1} \sum_{i_{2}=i_{1}}^{n-1} \sum_{i_{3}=i_{2}}^{n-1} x^{i_{3}}=\sum_{i_{2}=0}^{n-1} \sum_{i_{3}=i_{2}}^{n-1} x^{i_{3}}+\sum_{i_{2}=1}^{n-1} \sum_{i_{3}=i_{2}}^{n-1} x^{i_{3}}+\sum_{i_{2}=2}^{n-1} \sum_{i_{3}=i_{2}}^{n-1} x^{i_{3}}+\cdots+\sum_{i_{2}=n-1}^{n-1} \sum_{i_{3}=i_{2}}^{n-1} x^{i_{3}}$

$=\left(1+2 x+3 x^{2}+\cdots+n x^{n-1}\right)+\left(x+2 x^{2}+3 x^{3} \cdots+(n-1) x^{n-1}\right)+\cdots x^{n-1}$

$=1+3 x+6 x^{2}+10 x^{3}+\cdots+\frac{n(n+1)}{2 !} x^{n-1}=\sum_{i=0}^{n-1} \frac{(i+1)(i+2)}{2 !} x^{i}=\sum_{i=0}^{n-1} V_{i}^{2} x^{i}$

where $\sum_{i_{1}=0}^{n-1} \sum_{i_{2}=i_{1}}^{n-1} \sum_{i_{3}=i_{2}}^{n-1} x^{i_{3}}=1+3 x+6 x^{2}+10 x^{3}+15 x^{4}+21 x^{5}+\cdots+\frac{n(n+1)}{2 !} x^{n-1}$.

$\sum_{i_{1}=0}^{n-1} \sum_{i_{2}=i_{1}}^{n-1} \sum_{i_{3}=i_{2}}^{n-1} \sum_{i_{4}=i_{3}}^{n-1} x^{i_{4}}=\sum_{i=0}^{n-1} \frac{(i+1)(i+2)(x+3)}{3 !} x^{i}=\sum_{i=0}^{n-1} V_{i}^{3} x^{i}$

where $\sum_{i_{1}=0}^{n-1} \sum_{i_{2=i_{1}}} \sum_{i_{3}=i_{2}}^{n-1} \sum_{i_{4}=i_{3}}^{n-1} x^{i_{4}}=1+4 x+10 x^{2}+20 x^{3}+35 x^{4}+\cdots+\frac{n(n+1)(n+2)}{3 !} x^{n-1}$.

If we continue like this, the binomial coefficient of the multisereis is $V_{i}^{p}(1 \leq p \leq n-1)$. 


\section{To convert the combination $\mathrm{nCr}$ into the optimized combination:}

$n C r=\frac{n !}{r !(n-r) !}=\left(V_{0}^{r}\right)\left(V_{r}^{n-1}\right)=V_{r}^{n-r}$ where $V_{0}^{r}=1$.

Let us consider $n-r=k$ for easily understood.

Then, $\quad V_{r}^{n-r}=V_{r}^{k}=\frac{(r+1)(r+2)(r+3) \cdots(r+k)}{k !}$.

To convert the combination $n C n$ into the optimized combination:

$\mathrm{nCn}=\frac{n !}{n !}=V_{0}^{n}=1$.

To convert the combination $(\boldsymbol{n}+\boldsymbol{r}) \mathrm{Cr}$ into the optimized combination:

$(n+r) C r=\frac{n !}{r !(n+r-r) !}=\frac{n !}{r ! n !}=\frac{1.2 .3 \cdots r(r+1)(r+2) \cdots(r+n)}{r ! n !}=\left(V_{0}^{r}\right)\left(V_{r}^{n}\right)$.

$\left(V_{0}^{r}\right)\left(V_{r}^{n}\right)=V_{r}^{n}$ where $V_{0}^{r}=1$.

Now $V_{r}^{n}(n, r \in N, n \geq 1, \& r \geq 0)$ is considered as optimized combination.

Some results with proofs on the optimized combination [Annamalai, 2020] are provided below.

Result 1: $V_{0}^{1}=V_{0}^{n}=1$

Proof. $V_{0}^{1}=\frac{(0+1)}{1 !}=1$

$V_{0}^{n}=\frac{(0+1)(0+2)(0+3) \cdots \cdots(0+n)}{n !}=\frac{n !}{n !}=1$

From (i) and (ii), the result 1 is true.

Result 2: $\boldsymbol{V}_{r}^{n+1}-V_{r}^{n}=V_{r-1}^{n}$

Proof. $V_{r}^{n}=\frac{(r+1)(r+2)(r+3) \cdots \cdots(r+n)}{n !}$

$V_{r}^{n+1}=\frac{(r+1)(r+2)(r+3) \cdots \cdots(r+n)(r+n+1)}{(n+1) !}$

$V_{r}^{n+1}-V_{r}^{n}=\frac{(r+1)(r+2)(r+3) \cdots \cdots(r+n)}{n !}\left[\frac{r+n+1}{n+1}-1\right]$

$V_{r}^{n+1}-V_{r}^{n}=\frac{r(r+1)(r+2(r+3) \cdots \cdots(r+n)}{n !}=V_{r-1}^{n}$

It is understood from (iii) that the result 2 is true.

Result 3: $1+V_{1}^{1}+V_{1}^{2}+V_{1}^{3} \cdots \cdots V_{1}^{n}=V_{2}^{n}$

Proof. $V_{2}^{n}=\frac{(2+1)(2+2)(2+3) \cdots \cdots(2+n-1)(2+n)}{n !}=\frac{(n+1)(n+2)}{2 !}$

$1+V_{1}^{1}+V_{1}^{2}+V_{1}^{3} \cdots \cdots V_{1}^{n}=1+2+3+\cdots+n+1=\frac{(n+1)(n+2)}{2 !}$

From (iv) and (v), the result 3 is true.

Result 4: $V_{r}^{n}=V_{n}^{r}(n, r \geq 1 \& n, r \in N)$

Proof. $V_{r}^{n}=V_{n}^{r}$ implies $\frac{(r+1)(r+2) \cdots(r+n)}{n !}=\frac{(n+1)(n+2) \cdots(n+r)}{r !}$ 
Assume that $r=n+m(m \in N \& m \geq 1)$. Let us show that $V_{n+m}^{n}=V_{n}^{n+m}$.

$V_{n+m}^{n}=\frac{(n+m+1)(n+m+2) \cdots(n+m+n)}{n !}=\frac{(n+1)(n+2) \cdots(n+m+n)}{(n+m) !}$

$V_{n}^{n+m}=\frac{(n+1)(n+2) \cdots(n+n)(n+n+1)(n+n+2) \cdots(n+n+m)}{(n+m) !}$

From (vi)and (vii), $\quad V_{n+m}^{n}=V_{n}^{n+m}$ is true.

Assume that $r=n-m(n>m)$. Let us show that $V_{n-m}^{n}=V_{n}^{n-m}$.

$V_{n-m}^{n}=\frac{(n-m+1)(n-m+2) \cdots(n-m+n)}{n !}=\frac{(n+1)(n+2) \cdots(n+n-m)}{(n-m) !}$

$V_{n}^{n-m}=\frac{(n+1)(n+2) \cdots(n+n-m)}{(n-m) !}$

From (viii))and (ix), $V_{n-m}^{n}=V_{n}^{n-m}$ is true.

If $r=n, V_{r}^{n}=V_{n}^{r}$ is obivously true for $r=n$.

Hence, the result 4 is true.

Result 5: $V_{n}^{n}=2 V_{n-1}^{n}$

Proof. $V_{n}^{n}=\frac{(n+1)(n+2) \cdots(n+n-1) 2 n}{(n-1) ! n}=\frac{2(n+1)(n+2) \cdots(n+n-1)}{(n-1) !}=2 V_{n-1}^{n}$

Hence, the result 5 is true.

Result 6: $V_{0}^{n}+V_{1}^{n}+V_{2}^{n}+V_{3}^{n} \cdots+V_{r-1}^{n}+V_{r}^{n}=V_{r}^{n+1}$

Proof. This result is proved by mathematical induction.

Basis. Let $r=1 . V_{0}^{n}+V_{1}^{n}=V_{1}^{n+1}$ implies $n+2=n+2$.

Inductive hypothesis.

Let us assume that $V_{0}^{n}+V_{1}^{n}+V_{2}^{n}+\cdots+V_{k-1}^{n}=V_{k-1}^{n+1}$ is true for $r=k-1$.

Inductive step. We must show that the inductive hypothesis is true for $\mathrm{r}=\mathrm{k}$.

$V_{0}^{n}+V_{1}^{n}+\cdots+V_{k-1}^{n}+V_{k}^{n}=V_{k}^{n+1}$ implies $V_{0}^{n}+V_{1}^{n}+\cdots+V_{k-1}^{n}=V_{k}^{n+1}-V_{k}^{n}=V_{k-1}^{n+1}$

Hence, it is proved.

\section{CONCLUSION}

In the research paper, optimized combination of combinatorics has been introduced that are useful for scientific researchers who are solving scientific problems and meeting today's challenges. The optimized combination was derived from the recursive computation of multgeometric series and summability. The combinatorial computing technique, called optimized combination, has been applied in differential and integral equation developed by using multiple geometric series and summability.

\section{R E F E R E N C E S}

Annamalai, C., 2018. Annamalai's Computing Model for Algorithmic Geometric series and Its Mathematical Structures. Mathematics and Computer Science - Science Publishing Group, USA. 3(1) 1-6.
Annamalai, C., 2018, Algorithmic Computation for Annamalai's Geometric Series and Summability. Mathematics and Computer Science - Science Publishing Group, USA. 3(5) 100-101.

Annamalai, C., 2019, A Model of Iterative Computations for Recursive Summability. Tamsui Oxford Journal of Information and Mathematical Sciences - Airiti Library. 33(1) $75-77$.

Annamalai, C., Srivastava, H. M., Mishra, V. N., 2020, Recursive Computations and Differential and Integral Equations for Summability of Binomial Coefficients with Combinatorial Expressions. International Journal of Scientific Research in Mechanical and Materials Engineering. 4(1)1-5.

Annamalai, C., 2020, Novel Computing Technique in combinatorics. Archive ouverte HAL. https://hal.archivesouvertes.fr/hal-02862222

Annamalai, C., 2020, Optimized Computing Technique for Combination in Combinatorics. Archive ouverte HAL. https://hal.archives-ouvertes.fr/hal-02865835 